\title{
Cratering rate on Pluto produced by the inner trans-Neptunian population
}

\author{
M. F. Calandra ${ }^{1}$ and R. Gil-Hutton ${ }^{1,2}$ \\ ${ }^{1}$ Grupo de Ciencias Planetarias, Complejo Astronómico El Leoncito, UNLP, UNC, UNSJ, CONICET, Av. España 1512 sur, \\ J5402DSP San Juan, Argentina \\ e-mail: fcalandra@casleo.gov.ar \\ 2 Universidad Nacional de San Juan, J. I. de la Roza 590 oeste, 5400 Rivadavia, San Juan, Argentina
}

Received 13 May 2016 / Accepted 4 February 2017

\begin{abstract}
Aims. The aim of this work is to obtain the cratering rate on Pluto and to estimate the size distribution of the population in the inner trans-Neptunian region.

Methods. We find the intrinsic collisional probability and the mean collision velocity for the interaction between Pluto and the projectile population crossing its orbit, using the L7 Synthetic Model from the CFEPS Project. The size distribution of this population is found using the smallest satellite of Pluto, Styx, as a constraint, because it survives the collisional process for the solar system age. Results. We find that the mean intrinsic collisional probability and mean collision velocity between Pluto and the projectile population are $\left\langle P_{i}\right\rangle=1.3098 \times 10^{-22} \mathrm{~km}^{-2} \mathrm{yr}^{-1}$ and $\left\langle V_{\text {col }}\right\rangle=2.005 \pm 0.822 \mathrm{~km} \mathrm{~s}^{-1}$. If the projectile sample is separated between Plutinos and non-Plutinos and the intrinsic collisional probability of these sub-populations are taken into account, we find a ratio of approximately 20:1 in favor of non-Plutinos resulting in the greatest contribution to the cratering rate on Pluto. The projectile population for the inner trans-Neptunian belt is characterized using a double power-law mean-size distribution with exponents $q_{A}=3.5$ and $q_{B}=5.14$ for the small and large size end of the population, respectively, and break radius at $r_{\mathrm{b}}=11.86 \mathrm{~km}$ or $7.25 \mathrm{~km}$ for mean densities of the projectiles $\rho_{1}=1.85 \mathrm{~g} \mathrm{~cm}^{-3}$ and $\rho_{2}=1 \mathrm{~g} \mathrm{~cm}^{-3}$. With this mean-size distribution we find that an object with radius of $\sim 28 \mathrm{~km}$ produces a crater in Pluto with a diameter of $\sim 250 \mathrm{~km}$ in a time larger than the solar system age, indicating that this kind of large structure has a very low probability of occurrence.
\end{abstract}

Key words. Kuiper belt: general - methods: numerical - planets and satellites: general

\section{Introduction}

Pluto is the largest and second-most-massive known dwarf planet in the solar system, located in the inner region of the trans-Neptunian belt. Pluto with its largest satellite, Charon, was until recently considered the binary object with the largest primary-to-secondary mass ratio in the solar system. Recently, four new small satellites were discovered using the Hubble Space Telescope: Nix and Hydra in 2005, Kerberos in 2011, and Styx in 2012. The discovery of these small objects changed the model that is used to explain the formation of the satellite system around Pluto, which is based on a scenario similar to that proposed for the formation of the Earth-Moon system (Canup \& Asphaug 2001; Canup 2004).

The current model of the Pluto-Charon system argues in favor of a giant impact wherein a binary system was formed from an oblique low-velocity collision between the planet and a protosatellite (McKinnon 1989; Canup 2005) and in which Charon arose from the reaccumulation of material on an intact portion of the impactor. The discovery of the small satellites led Canup (2011) to study the new scenario and find that it is possible to form an intact Charon and the smaller satellites simultaneously from the debris produced by a single impact.

Nevertheless, it is also important to study how the role of the collisional process and its effects in the inner zone of the transNeptunian belt could affect the objects in this region. Both Pluto and its satellites show craters on their surfaces that are the result of the collisional activity.

Weissman \& Stern (1994) were two of the first authors that studied the impact rate onto Charon and Pluto, motivated by the improvement in knowledge of the radii and masses of the first trans-Neptunian objects. They found that objects with diameters $d>2.4 \mathrm{~km}$ could collide with Pluto in $1.9 \mathrm{Myr}$ and with Charon in $10 \mathrm{Myr}$. On the other hand, for the same impactor size range and collisional process, Durda \& Stern (2000) found timescales of $0.39 \mathrm{Myr}$ and 3.2 Myr for Pluto and Charon, respectively. These seem to be an overestimation of the impact rate produced by an incorrect evaluation of the projectile population size distribution. Later, Zahnle et al. (2003) studied the cratering rate in the outer solar system using two different size distributions and found values of $1 \times 10^{-6} \mathrm{yr}^{-1}$ and $2.6 \times 10^{-6} \mathrm{yr}^{-1}$ for Pluto's collisions with objects larger than $1.5 \mathrm{~km}$.

Recently, de Elía et al. (2010) studied the interaction between Pluto and the population of Plutinos using numerical simulations and found that these objects strike Pluto on timescales of 2.27 to $9.9 \mathrm{Myr}$, and proposed that this could be the population that dominate the collisional process on Pluto. However, Bierhaus \& Dones (2015) argued against this conclusion because they found that the impact flux on this dwarf planet is not dominated by the Plutinos, suggesting that the projectiles impacting on Pluto come mainly from another population. Bierhaus \& Dones (2015) found that the cratering rates for Pluto 
are $6.7 \times 10^{-9} \mathrm{yr}^{-1}$ and $4.6 \times 10^{-7} \mathrm{yr}^{-1}$, considering projectiles with radius larger than $5 \mathrm{~km}$ and $0.5 \mathrm{~km}$, respectively. Finally, Greenstreet et al. (2015) modeled the size distribution for the impactor population using different power-laws and found a cratering rate on Pluto in the range $5 \times 10^{-7}$ to $9 \times 10^{-8} \mathrm{yr}^{-1}$ for projectiles with radius larger than $5 \mathrm{~km}$.

In all of these works the largest uncertainty in computing the impact rates on Pluto arises from the extrapolation in size from the known largest objects to the unknown smallest objects needed to define the projectile size distribution. The knowledge of the population size distribution in the inner region of the trans-Neptunian belt could provide clues about the giant-planet formation process and the early solar system environment (Davis \& Farinella 1997; Gladman et al. 1998; Kenyon \& Bromley 2004). It also offers valuable information about the evolutionary processes, the differences between the size distribution in different regions of the trans-Neptunian belt, and how these size distributions affect those populations (Weissman et al. 2009; Fraser et al. 2008).

Recently, NASA's spacecraft New Horizons flew over Pluto in July 2015. It obtained important information about the sizes of the objects in the Pluto system and found features on their surfaces indicating an intense collisional process. The Pluto radius determined by New Horizons is similar to that found from Earth by means of stellar occultations $(1190 \pm 5 \mathrm{~km}$, Dias-Oliveira et al. 2015). The spacecraft also provided images of the small satellites that were used to produce some good estimations for their sizes. Despite the intense collisional activity that modified the surfaces of Pluto and Charon, this process did not destroy the small satellites by means of a catastrophic collision. This fact can be used as a threshold to study the size distribution of the projectile population in the inner trans-Neptunian belt.

In this paper we present an estimation of the size distribution for the population of small bodies crossing the orbit of Pluto and the resulting collisional rate on this dwarf planet. We describe the method used for the selection process and simulations in Sect. 2. In Sect. 3 we discuss our results and in Sect. 4 we summarize the conclusions.

\section{Methods}

In order to determine the size distribution of the population crossing Pluto's orbit it is necessary to begin considering the probability of collision $p$ of a population of projectiles with a certain target:

$p=\left\langle P_{i}\right\rangle \tau^{2} \Delta t N\left(>r_{p}\right)$,

where $\left\langle P_{i}\right\rangle$ is the mean intrinsic collisional probability of the target; $\tau=R_{t}+r_{p}$ is the geometric cross section; $R_{t}$ and $r_{p}$ are the radius of the target and the projectile, respectively; and $N\left(>r_{p}\right)$ is the number of projectiles with radius larger than $r_{p}$. This equation implies that if $p=1$ the target receives one collision by an object with radius $r_{p}$ in a time span $\Delta t$ and under those conditions we can estimate $N\left(>r_{p}\right)$, which is a function of the projectile population size distribution.

The mean collision velocity and mean intrinsic collisional probability of Pluto can be obtained using the method developed by Marzari et al. (1996). In this method the target and the projectile population are numerically integrated during a time span $\Delta t$ and the encounter distance and velocity between the target and any projectile are recorded. Once the list of encounters is obtained, the accumulative number of encounters $N(d<D)$ is

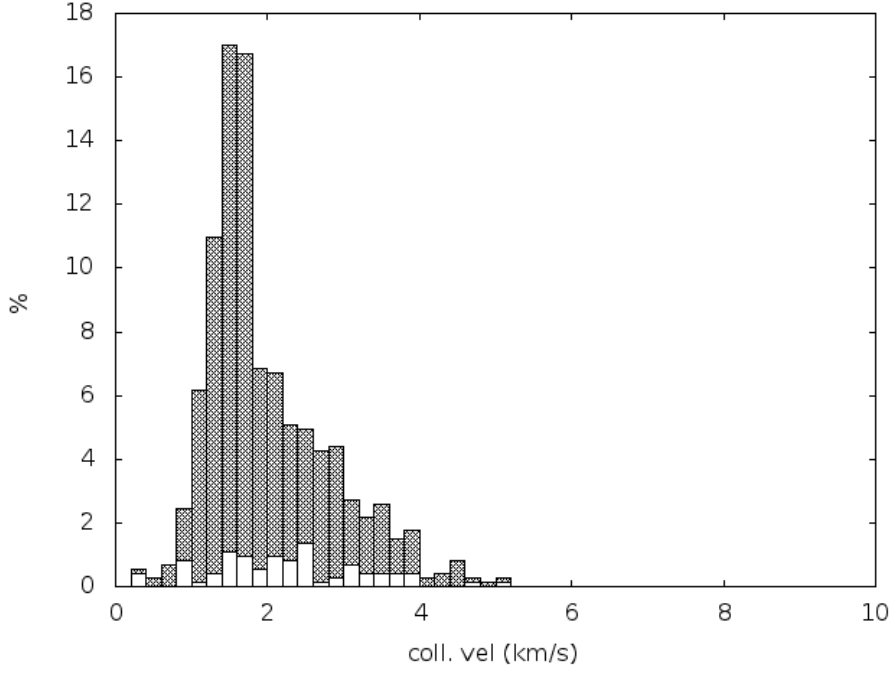

Fig. 1. Histogram of the collision velocities between Pluto and the projectile sample. The gray histogram is for the total sample and the white histogram is for the Plutino subsample.

calculated where $d$ is the approach distance and $D$ is a reference value. Because the cross-section is proportional to $\pi d^{2}$ the accumulative number of encounters is $N(d<D)=P_{1} D^{2}$ where $P_{1}$ is a constant that is found by a fit to the data. The mean intrinsic collisional probability is related with $P_{1}$ through the expression:

$\left\langle P_{i}\right\rangle=\frac{P_{1}}{n_{\text {pair }} \Delta t}$,

where $n_{\text {pair }}$ is the total number of target-projectile pairs.

To apply the method of Marzari et al., we need orbital elements of the objects that cross the orbit of Pluto. Because the L7 Synthetic Model from the Canada France Ecliptic Plane Survey (CFEPS) $)^{1}$ (Kavelaars et al. 2009) is a debiased model for the TNO population down to $H_{g}=8.5 \mathrm{mag}$, we used it to extract the objects with aphelia greater than 29.7 au and perihelia less than $49.3 \mathrm{au}$.

Using this sample of the inner trans-Neptunian belt we made a synthetic population of 4950 objects that follows the orbital element distribution obtained from the L7 model. The dynamical evolution of this projectile population was followed during $5 \times 10^{6}$ yr by a numerical integration using the symplectic code EVORB (Fernández et al. 2002) with a time step of $0.1 \mathrm{yr}$, and including Pluto and the four giant planets as perturbers. In each time-step the code searches for encounters between Pluto and the projectile population and at the end of the integration 730 encounters were found for 4948 objects that survive the integration period.

Using the synthetic population and applying the method of Marzari et al. (1996) we calculated the current mean intrinsic collisional probability and mean collision velocity for Pluto, obtaining $\left\langle P_{i}\right\rangle=1.3098 \times 10^{-22} \mathrm{~km}^{-2} \mathrm{yr}^{-1}$ and $\left\langle V_{\text {col }}\right\rangle=2.005 \pm$ $0.822 \mathrm{~km} \mathrm{~s}^{-1}$. The distribution of collisional velocities between Pluto and the projectile population is shown in Fig. 1. The peak in the distribution at $V_{\text {col }} \sim 1.8 \mathrm{~km} \mathrm{~s}^{-1}$ is produced by nonPlutinos reaching their aphelion approaching Pluto with very low velocity. The tail of high collision-velocities could be produced by projectiles with high eccentricity or inclination, indicating that different populations are involved in the collisional process on Pluto.

\footnotetext{
1 ftp:http://wwW.cfeps.net/tnodb/ the-17-synthetic-model/
} 
Table 1. Number of objects $(N), P_{1},\left\langle P_{i}\right\rangle,\left\langle V_{\text {col }}\right\rangle$, and the number of encounters with Pluto for the Plutino and non-Plutino sub-samples.

\begin{tabular}{lll}
\hline \hline & Plutinos & non-Plutinos \\
\hline$N$ & 235 & 4715 \\
$P_{1}$ & 8444.42 & 64511.7 \\
$\left\langle P_{i}\right\rangle$ & $3.225 \times 10^{-22}$ & $1.223 \times 10^{-22}$ \\
$\left\langle V_{\text {col }}\right\rangle$ & $2.265 \pm 0.988$ & $1.975 \pm 0.795$ \\
Encounters & 77 & 653 \\
\hline
\end{tabular}

Notes. $\left\langle P_{i}\right\rangle$ and $\left\langle V_{\text {col }}\right\rangle$ are in units of $\mathrm{km}^{-2} \mathrm{yr}^{-1}$ and $\mathrm{km} \mathrm{s}^{-1}$, respectively.

The contribution of the different populations can be analyzed dividing the sample into at least two sub-samples according to whether the object is a Plutino or not. The values of $\left\langle P_{i}\right\rangle$ and $\left\langle V_{\text {col }}\right\rangle$ for these sub-samples were calculated using the same procedure applied to the total sample and are shown in Table 1. In the case of the Plutinos this result agrees very well with that proposed by Dell'Oro et al. (2013), meaning that it confirms that the high collision-velocity tail observed in Fig. 1 is a consequence of the interaction of Pluto with the non-Plutino population. Despite the fact that the intrinsic collisional probability of Pluto with the Plutinos is approximately 2.5 times that of non-Plutinos, the non-Plutino group is almost 20 times larger, producing 10 times more encounters with Pluto and dominating the collisional rate on it. This result does not agree with de Elía et al. (2010), who propose that the Plutinos could be the population that dominates the collisional process on Pluto. However, the result does agree with Bierhaus \& Dones (2015), who study the cratering rate on Pluto and Charon and argues that the impact flux come mainly from another population.

As we mention in the introduction, one important constraint for the collisional process in the Pluto system is that Styx survives the collisional process during the solar system age in spite of the fact that its size is very small. Showalter \& Hamilton (2015) found a radius of $\sim 2.1 \mathrm{~km}$ for this small satellite, but in a recent work Weaver et al. (2016) obtained more accurate values for the sizes of Pluto's moons, proposing a radius of $\sim 5.2 \mathrm{~km}$ for Styx. Using the latter value for the radius we can find the minimum projectile size necessary to destroy Styx by using the expression proposed by Benz \& Asphaug (1999) to calculate the specific energy required to break a body into fragments:

$Q_{D}^{*}=Q_{0} r_{t}^{a}+B \rho r_{t}^{b}$,

where $r_{t}$ is the target radius and we have for ice $Q_{0}=1.6 \times$ $10^{7} \mathrm{erg} \mathrm{s}^{-1}, B=1.2 \mathrm{erg} \mathrm{cm}^{3} \mathrm{~g}^{-2}, a=-0.39$ and $b=1.26$, whereas for basalt $Q_{0}=3.5 \times 10^{7} \mathrm{erg} \mathrm{s}^{-1}, B=0.3 \mathrm{erg} \mathrm{cm}^{3} \mathrm{~g}^{-2}$, $a=-0.38$ and $b=1.36$. In both cases $\rho$ is the density of the target in $\mathrm{g} \mathrm{cm}^{-3}$. Therefore the energy balance is:

$Q_{D}^{*} M_{t}=Q m_{p}$

where $Q$ is the specific kinetic energy of the projectile. Using these expressions we can calculate the projectile mass and radius by assuming that the collision velocity is $\left\langle V_{\text {col }}\right\rangle$ and the target has a density of $\rho=1.85 \mathrm{~g} \mathrm{~cm}^{-3}$, which is the density for Pluto found by Dias-Oliveira et al. (2015). For the projectile we used two different values for this parameter: $\rho_{1}=1.85 \mathrm{~g} \mathrm{~cm}^{-3}$ equal to the density of the target, and $\rho_{2}=1 \mathrm{~g} \mathrm{~cm}^{-3}$ which corresponds to a typical density of a binary trans-Neptunian object. Using these values, the projectile radius able to destroy a target with a radius of $5.2 \mathrm{~km}$ is $r_{p 1}=0.578 \mathrm{~km}$ and $r_{p 2}=0.710 \mathrm{~km}$, respectively.

These results can be used in Eq. (1) to obtain the mean number of collisions received by Styx or how long it must wait to receive at least one destructive collision. Then, using a time interval $\Delta t$ equal to the solar system age $\left(4.6 \times 10^{9} \mathrm{yr}\right)$, the value for $N\left(>r_{p}\right)$ can be found if we know the size distribution of the projectile population. This size distribution can be modeled assuming a single power law of the form:

$\mathrm{d} N_{\text {pro }}(>r)=K r^{-q} \mathrm{~d} r$

where $r$ is the projectile radius, $q$ is a characteristic exponent of the single power-law size distribution, and $K$ is a proportionality constant. In order to find the parameters $K$ and $q$, it is necessary to perform a least-squares fit to the synthetic sample obtained from L7. A value of $q=5.14$ was found for the exponent of the size distribution and the proportionality constant was $K=2.426629 \times 10^{11}$.

This size distribution for the projectile population and Eq. (1) indicate that Styx should wait $4.23 \times 10^{8} \mathrm{yr}$ or $9.48 \times 10^{8} \mathrm{yr}$ to receive at least one destructive collision for $r_{p 1}=0.578 \mathrm{~km}$ or $r_{p 2}=0.710 \mathrm{~km}$, respectively. If the assumption of a single power-law size distribution is correct, this result indicates that Styx was formed or arrived to the Pluto system recently. However, if we accept that it was old and formed at the same time of Charon because it has not yet been destroyed it is necessary to obtain a smaller value for $N\left(>r_{p}\right)$, suggesting a projectile population size distribution which could be modeled by a double power law. This would allow a reduction in the number of projectiles that could destroy Styx in a period equal to the solar system age.

Assuming that Styx formed early and that the size distribution could change for different size ranges, the number of projectiles with radius greater than $r_{p}$ can be represented by using a double power-law:

$N_{\text {pro }}\left(>r_{p}\right)=K_{A} \int_{r_{p}}^{r_{\mathrm{b}}} r^{-q_{A}} \mathrm{~d} r+K_{B} \int_{r_{\mathrm{b}}}^{r_{1}} r^{-q_{B}} \mathrm{~d} r$,

where $K_{A}$ and $K_{B}$ are proportionality constants, $r_{1}$ is the radius of the largest object in the population, and $r_{\mathrm{b}}$ is the break radius of the size distribution for two arbitrary size ranges with exponents $q_{A}$ and $q_{B}$, respectively.

To find the value of the radius at which the size distribution changes, we assumed that the large end of the projectile population follows the single power-law previously found, and that $K_{B}=K$ and $q_{B}=q$. In the case of the small end of the population we assumed $q_{A}=3.5$, which is the exponent proposed by Dohnanyi $(1969,1971)$ for a steady-state population. Thus, assuming as a constraint that Styx received less than one catastrophic collision $(p<1)$ in a time interval $\Delta t=4.6 \times 10^{9} \mathrm{yr}$, we found $K_{A}=3.313341 \times 10^{10}$ and $r_{\mathrm{b}}=2.4757 \mathrm{~km}$ for $r_{p 1}$, and $K_{A}=5.296306 \times 10^{10}$ and $r_{\mathrm{b}}=1.8599 \mathrm{~km}$ for $r_{p 2}$.

The size distribution of the projectile population was obtained assuming a population that is equal to the present one and it does not change over time. However, the number of solar system objects in the past must be larger than in the present and the mean probability to receive a catastrophic collision during the solar system age is also larger than that we just obtained. Therefore a re-calculation that takes the time evolution of the population into account was necessary.

As shown by several authors (Dohnanyi 1969; Farinella et al. 1985; Hellyer 1970), if the number of objects larger than $r$ at an epoch $t$ can be expressed as $C(t) N(t)$, the function $C(t)$ has the form:

$C(t)=C(0)\left[1+\left(\frac{C(0)}{C(\Delta t)}-1\right) \frac{t}{(\Delta t)}\right]^{-1}$, 
where $C(0)$ is the value at $t=0$ and $C(\Delta t)$ is the value at the present time. This function gives the time evolution of the population and it must be integrated and promediated over the solar system age to get a correction factor $\alpha$ :

$$
\alpha=\frac{1}{\Delta t C(\Delta t)} \int_{0}^{\Delta t} C(t) \cdot \mathrm{d} t=\left[1-\frac{C(\Delta t)}{C(0)}\right]^{-1} \ln \left(\frac{C(0)}{C(\Delta t)}\right) .
$$

Using this correction factor, Eq. (1) takes now the form:

$$
p=\left\langle P_{i}\right\rangle \tau^{2} \Delta t N_{\text {pro }}\left(>r_{p}\right) \alpha \text {. }
$$

Considering recent studies of the dynamical structure of the trans-Neptunian belt (Petit et al. 2011; Gladman et al. 2012) and according with the CFEPS survey, there is a probability of capture in the Plutino region of $P_{3: 2} \sim 1.3$ to $1.5 \times 10^{4}$ in a period equal to the solar system age (Nesvorný \& Vokrouhlický 2016). Using this value we find that $C(0) / C(\Delta t) \approx \frac{1}{P_{3 \cdot 2}} \sim 6700$ for the 3:2 resonant population, resulting in a correction factor of $\alpha \sim 8.8$ for the inner trans-Neptunian region. With this value it was possible to calculate a mean-size distribution for the projectile population by assuming: $p<1$ for Styx that is characterized by $K_{B}=2.1354 \times 10^{13}$; parameters $K_{A}=3.3133 \times 10^{10}$ and $r_{\mathrm{b}}=11.8606 \mathrm{~km}$ for $r_{p 1}$; and $K_{A}=5.296306 \times 10^{10}$ and $r_{\mathrm{b}}=7.2538 \mathrm{~km}$ for $r_{p 2}$.

\section{Results}

Using a reformulation of Eq. (1), the projectile size distribution found and the results obtained in the previous section allow us to calculate the cratering rate on Pluto produced by projectiles crossing Pluto's orbit. If we now consider Pluto as a target, it is necessary to take into account the effects of gravitational focusing that increase the geometrical section by a factor $\Gamma=1+\left(v_{\text {esc }} / v_{\infty}\right)^{2}$, where $v_{\text {esc }}$ is the escape velocity for Pluto and $v_{\infty}$ is the relative velocity between Pluto and the projectile. For $v_{\infty}$ we take the value of the mean collisional velocity obtained above and obtain $\Gamma=1.2951$. This result is similar to the value found by Bierhaus \& Dones (2015), which is a consequence of the good agreement between our mean collision velocity and the collision velocity calculated by Dell'Oro et al. (2013).

Then, using the mean-size distribution parameter $K_{A}$ for $r_{\mathrm{b}}=7.2538 \mathrm{~km}$ and projectiles radii of $5 \mathrm{~km}, 1.5 \mathrm{~km}$ and $0.5 \mathrm{~km}$, to allow a comparison of our results with other authors, the mean cratering rate on Pluto corrected by gravitational focusing is $\langle C R\rangle \simeq 8.746 \times 10^{-8} \mathrm{yr}^{-1},\langle C R\rangle \simeq 1.763 \times 10^{-6} \mathrm{yr}^{-1}$ and $\langle C R\rangle \simeq 2.745 \times 10^{-5} \mathrm{yr}^{-1}$, respectively. These results agree fairly well with those of Bierhaus \& Dones (2015) and Greenstreet et al. (2015). However, the differences between our work and theirs might arise from the difference in process used by these authors to evaluate the size distribution of the population. Bierhaus \& Dones and Greenstreet et al. made extrapolations from an assumed size distribution for the largest transNeptunian objects down to those of sub-kilometer sizes, whereas we used the present projectile population and a constraint on the survival of Styx.

The cratering rate found in this work allows us to estimate the age of the largest crater observed by the New Horizons spacecraft on Pluto, a structure with a diameter of $\sim 250 \mathrm{~km}$ (Moore et al. 2016). The size of the projectile that produced this crater can be calculated with the cratering scaling law proposed by Zahnle et al. (2003):

$D_{s}=13.4\left(\frac{V^{2}}{g}\right)^{0.217}\left(\frac{\rho}{\rho_{\text {Plu }}}\right)^{0.333}\left(\frac{d_{\mathrm{p}}}{\mathrm{km}}\right)^{0.783}(\cos \theta)^{0.333}$, and,

$D=\left\{\begin{aligned} D_{\mathrm{s}}, & \text { for } D_{\mathrm{s}} \leq D_{\mathrm{c}} \\ D_{\mathrm{s}}\left(\frac{D_{\mathrm{s}}}{D_{\mathrm{c}}}\right)^{\zeta}, & \text { for } D_{\mathrm{s}}>D_{\mathrm{c}}\end{aligned}\right.$

where $D$ is the crater diameter in $k m, D_{s}$ is the diameter of a simple crater in $\mathrm{km}, V$ is the collision velocity in $\mathrm{km} \mathrm{s}^{-1}, g$ is the gravitational acceleration on the surface of Pluto in $\mathrm{cm} \mathrm{s}^{-2}, \rho$ is the impactor density, $\rho_{\text {Plu }}$ is Pluto's density, $d_{\mathrm{p}}$ is the projectile diameter in $\mathrm{km}, \theta$ is the incident angle measured from the normal to the surface, $\zeta=0.13$ (McKinnon et al. 1991), and $D_{\text {c }}$ is the transition diameter between simple and complex craters. Assuming for Pluto $D_{\mathrm{c}}=6 \mathrm{~km}, g=64 \mathrm{~cm} / \mathrm{s}^{2}, V=\left\langle V_{\mathrm{col}}\right\rangle, \theta=45^{\circ}$, and using the same value for the densities of both Pluto and the projectile, a crater with this diameter could be produced by a collision with a projectile of $r_{\mathrm{p}}=28.39 \mathrm{~km}$. Considering the mean-size population obtained above, a crater of this size would be produced in a time longer than the solar system age. If $\theta, \rho$, or $V$ changes a little they do not produce a different result and the time needed to receive this sort of a collision is always larger than $\sim 4 \times 10^{9} \mathrm{yr}$.

Using the collisional rate on Pluto proposed here, a big crater such as the Burney Crater $(\sim 250 \mathrm{~km})$ needs a period longer than the solar system age to be produced. This implies that the probability of occurrence for these sorts of structures is very low and could be the result of an intense bombardment produced immediately after its formation due to a projectile population several times the present one. However, it is difficult to date this structure because there are several geological processes acting that have the ability to significantly change the surface of Pluto (Moore et al. 2016).

On the other hand, Weaver et al. (2016) studied the surfaces of Pluto's small satellites using images obtained by the New Horizons mission. In particular, these authors find several features on the surface of Nix, including an elongated object with size $50 \times 35 \times 33 \mathrm{~km}$ and a high albedo of $0.56 \pm 0.05$, which could indicate that this satellite is covered by water ice. Analyzing a surface of $1000 \mathrm{~km}^{2}$ on Nix, they identify 11 features that could be attributed to impact craters with diameters $>1.7 \mathrm{~km}$. Considering only the six craters with diameters in the range $4-14 \mathrm{~km}$ and using the mean-size distribution obtained above, these features must be the result of impacts with projectiles with $0.07 \leq r_{\mathrm{p}} \leq 0.213 \mathrm{~km}$ in a time interval of $2.672 \times 10^{7}$ to $2.147 \times 10^{8} \mathrm{yr}$. This result indicates that the surface of this satellite could be under a continuous resurfacing process that buried the older craters under the new ones, a process that could be favored by the presence of water ice on its surface.

\section{Conclusions}

In order to obtain the cratering rate on Pluto, we present an estimate of the size distribution for the population of small bodies crossing the orbit of this dwarf planet. The mean intrinsic collisional probability and mean velocity of collision of Pluto with the projectile population are $\left\langle P_{i}\right\rangle=1.3098 \times 10^{-22} \mathrm{~km}^{-2} \mathrm{yr}^{-1}$ and $\left.V_{\text {col }}\right\rangle=2.005 \pm 0.822 \mathrm{~km} \mathrm{~s}^{-1}$. If the sample is separated between Plutinos and non-Plutinos we find $\left\langle P_{i}\right\rangle=3.225 \times$ $10^{-22} \mathrm{~km}^{-2} \mathrm{yr}^{-1}$ and $\left\langle V_{\text {col }}\right\rangle=2.265 \pm 0.988 \mathrm{~km} \mathrm{~s}^{-1}$ for Plutinos, and $\left\langle P_{i}\right\rangle=1.223 \times 10^{-22} \mathrm{~km}^{-2} \mathrm{yr}^{-1}$ and $\left\langle V_{\text {col }}\right\rangle=1.975 \pm$ $0.7985 \mathrm{~km} \mathrm{~s}^{-1}$ for non-Plutinos. The result we find for Plutinos is in good agreement with the values obtained by Dell'Oro et al. (2013) and shows that the contribution from Plutinos and nonPlutinos are almost the same because the number of non-Plutino 
objects is compensated with a lower intrinsic collisional probability, and viceversa.

Taking into account the ratio between the sub-populations, we made and characterize a final projectile population using a single power-law size distribution with exponent $q=5.14$. However, we find that with this size distribution Styx received at least one destructive collision in $4.23 \times 10^{8} \mathrm{yr}$ and $9.48 \times 10^{8} \mathrm{yr}$ for the two projectile densities used. Thus, to obtain $p<1$ we change to a double power-law mean-size distribution with $q_{A}=3.5$ for the small size end of the population and break radius at $r_{\mathrm{b}}=11.8606 \mathrm{~km}$ or $r_{\mathrm{b}}=7.2538 \mathrm{~km}$ for mean densities $\rho_{1}=1.85 \mathrm{~g} \mathrm{~cm}^{-3}$ and $\rho_{2}=1 \mathrm{~g} \mathrm{~cm}^{-3}$, respectively.

With this mean-size population we calculate the cratering rate on Pluto for projectiles of different sizes and obtain results in agreement with Bierhaus \& Dones (2015) and Greenstreet et al. (2015). With these results we search for the projectile which produces the largest structure observed on Pluto (a crater with size $\sim 250 \mathrm{~km}$ ) and we find that an object with radius of $\sim 29 \mathrm{~km}$ produces a crater with that diameter in $\simeq 8 \times 10^{10} \mathrm{yr}$, indicating that these kinds of large structures have a low probability of occurrence on Pluto within the solar system age.

Finally, analyzing the six largest craters observed on Nix's surface and using the size distribution found for the projectile population, we find that these craters could be the result of impacts with objects with $0.07 \leq r_{\mathrm{p}} \leq 0.213 \mathrm{~km}$ in a short time of $2.672 \times 10^{7} \mathrm{yr}-2.147 \times 10^{8} \mathrm{yr}$. This indicates that the surface of this satellite could be subject to a continuous resurfacing process that erases the older craters by burying them under the new ones, a process that could be facilitated by the presence of water ice on its surface.

Acknowledgements. The authors thank the referee for the useful review, which led to a improvement of the paper. R.G.H. gratefully acknowledges financial support by CONICET through PIP 114-201101-00358.

\section{References}

Benz, W., \& Asphaug, E. 1999, Icarus, 142, 5

Bierhaus, E. B., \& Dones, L. 2015, Icarus, 246, 165

Canup, R. M. 2004, Icarus, 168, 433

Canup, R. M. 2005, Science, 307, 546

Canup, R. M. 2011, AJ, 141, 35

Canup, R. M., \& Asphaug, E. 2001, Nature, 412, 708

Davis, D. R., \& Farinella, P. 1997, Icarus, 125, 50

de Elía, G. C., di Sisto, R. P., \& Brunini, A. 2010, A\&A, 521, A23

Dell'Oro, A., Campo Bagatin, A., Benavidez, P. G., \& Alemañ, R. A. 2013, A\&A, 558, A95

Dias-Oliveira, A., Sicardy, B., Lellouch, E., et al. 2015, ApJ, 811, 53

Dohnanyi, J. S. 1969, J. Geophys. Res., 74, 2531

Dohnanyi, J. S. 1971, NASA Special Publication, 267, 263

Durda, D. D., \& Stern, S. A. 2000, Icarus, 145, 220

Farinella, P., Paolicchi, P., \& Zappala, V. 1985, MNRAS, 216, 565

Fernández, J. A., Gallardo, T., \& Brunini, A. 2002, Icarus, 159, 358

Fraser, W. C., Kavelaars, J. J., Holman, M. J., et al. 2008, Icarus, 195, 827

Gladman, B., Kavelaars, J. J., Nicholson, P. D., Loredo, T. J., \& Burns, J. A. 1998, AJ, 116, 2042

Gladman, B., Lawler, S. M., Petit, J.-M., et al. 2012, AJ, 144, 23

Greenstreet, S., Gladman, B., \& McKinnon, W. B. 2015, Icarus, 258, 267

Hellyer, B. 1970, MNRAS, 148, 383

Kavelaars, J. J., Jones, R. L., Gladman, B. J., et al. 2009, AJ, 137, 4917

Kenyon, S. J., \& Bromley, B. C. 2004, AJ, 128, 1916

Marzari, F., Scholl, H., \& Farinella, P. 1996, Icarus, 119, 192

McKinnon, W. B. 1989, ApJ, 344, L41

McKinnon, W. B., Chapman, C. R., \& Housen, K. R. 1991, Cratering of the Uranian satellites (Tucson: University of Arizona Press), 629

Moore, J. M., McKinnon, W. B., Spencer, J. R., et al. 2016, Science, 351, 1284

Nesvorný, D., \& Vokrouhlický, D. 2016, ApJ, 825, 94

Petit, J.-M., Kavelaars, J. J., Gladman, B. J., et al. 2011, AJ, 142, 131

Showalter, M. R., \& Hamilton, D. P. 2015, Nature, 522, 45

Weaver, H. A., Buie, M. W., Buratti, B. J., et al. 2016, Science, 351, aae0030

Weissman, P. R., \& Stern, S. A. 1994, Icarus, 111, 378

Weissman, P. R., Snodgrass, C., Fitzsimmons, A., \& Lowry, S. C. 2009, in

AAS/Division for Planetary Sciences Meeting Abstracts, 41, 2313

Zahnle, K., Schenk, P., Levison, H., \& Dones, L. 2003, Icarus, 163, 263 